\title{
Degenerated oocyte in the cohort adversely affects IVF outcome
}

\author{
Yuval Atzmon*, Mediea Michaeli, Diana Poltov, Nechami Rotfarb, Oshrit Lebovitz, Nardin Aslih and \\ Einat Shalom-Pazid
}

\begin{abstract}
The presence of Degenerated Oocyte (DEG) was mostly described after intracytoplasmic sperm injection (ICSI), with fewer reports on DEG at the time of ovum pick-up (OPU). This study aims to assess morphokinetics of embryos cultured in a time-lapse incubator and compare cohorts with and without DEG at OPU. In a retrospective cohort study from January 1, 2016 until September 31, 2017 a total of 399 IVF/ICSI cycles and 2980 embryos were evaluated. In 81 of 399 cycles at least one DEG oocyte was observed at the time of OPU. The remaining 318 cycles with no DEG oocyte were compared as a control group. In the DEG group, significantly more oocytes were collected per patient $(12.9 \pm 7.2$ vs. $10.1 \pm 6.1 . P<0.001)$. Fertilization rate, pregnancy and clinical pregnancy rates were comparable between the two groups, however, the morphokinetics and developmental scores of the embryos were significantly worse in the DEG group, (KID $3.4 \pm 1.6$ vs. $3.2 \pm 1.6 P=0.002$ and ESHRE $1.5 \pm 1.1$ vs. $1.4 \pm 1.0 P=0.046)$. Significantly more patients achieved top-quality embryos in the NON DEG group (58.8\% vs. $53.0 \%, P=0.03)$, however, comparable delivery rate was achieved in both groups. In the DEG group, the frequency of DEG oocyte per cycle was negatively correlated with pregnancy rate. GnRH agonist protocol and the 17-20G needle used for OPU were significant predictors for the presence of DEG oocyte at OPU. In conclusions DEG oocyte may negatively affect IVF outcome, however, younger patients, and significantly more oocytes collected in the DEG group compensate for the IVF results.
\end{abstract}

Keywords: Degenerated oocyte, Embryo morphokinetics, Ovum pick-up, Top-quality embryo, Aspiration needle

\section{Introduction}

The success rate of in vitro fertilization (IVF) depends on several parameters, of which good quality oocytes is the most important [1]. A degenerated (DEG) oocyte is described as an empty zona pellucida (EZP) or damaged oocyte (fragments of oocytes) within the zona pellucida [2]. These oocytes can be seen at the time of ovum pick-up (OPU) or after intracytoplasmic sperm injection (ICSI) (Fig. 1). To the best of our knowledge the presence of DEG oocytes immediately after OPU has not been previously studied as itis usually not reported by embryology labs. Oocyte quality before ICSI is very important and affects

\footnotetext{
*Correspondence: atzmony@gmail.com

IVF Unit, Department of Obstetrics and Gynecology, Hillel-Yaffe Medical Center, Hadera, Israel; affiliated with the Ruth and Bruce Rappaport School of Medicine, The Technion - Israel Institute of Technology, Haifa, Israel
}

treatment outcomes. The presence of EZP or DEG oocyte at OPU before ICSI correlates with the quality of the entire oocyte cohort [3-6]. Studies that examined the presence of EZP oocyte at OPU, found worse quality of oocytes collected in the same cycle, lower fertilization, poor embryo formation, and low pregnancy rates [2, 7]. Cinar et al. [2] reported worse performance of oocyte cohort when EZP oocyte were present in the aspirated group. Our study focused on DEG oocyte at the time immediately after OPU.

Currently, an automated time-lapse incubator allows for continuous and objective evaluation of fertilized oocytes and early embryo developmental morphokinetics [8], using the Known Implantation Data (KID) score which assigns morphokinetic parameters from 1 to 5 to estimate embryo viability and implantation potential [9]. Using embryo viewer software, kinetic markers are used

(c) The Author(s). 2020 Open Access This article is licensed under a Creative Commons Attribution 4.0 International License, which permits use, sharing, adaptation, distribution and reproduction in any medium or format, as long as you give appropriate credit to the original author(s) and the source, provide a link to the Creative Commons licence, and indicate if changes were made. The images or other third party material in this article are included in the article's Creative Commons licence, unless indicated otherwise in a credit line to the material. If material is not included in the article's Creative Commons licence and your intended use is not permitted by statutory regulation or exceeds the permitted use, you will need to obtain permission directly from the copyright holder. To view a copy of this licence, visit http://creativecommons.org/licenses/by/4.0/ The Creative Commons Public Domain Dedication waiver (http://creativecommons.org/publicdomain/zero/1.0/) applies to the data made available in this article, unless otherwise stated in a credit line to the data. 


\section{a. Damaged Oocyte (DEG) before denuding}

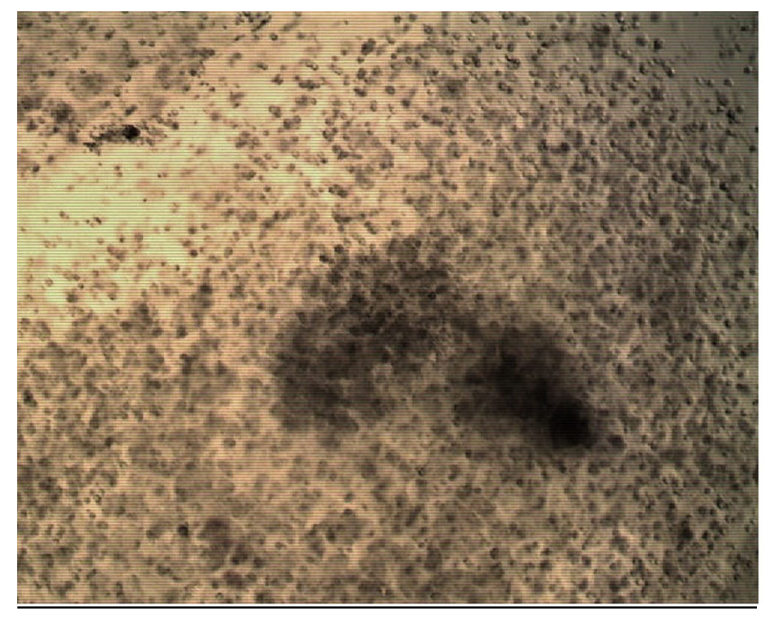

\section{b. Damaged Oocyte (DEG) after denuding}

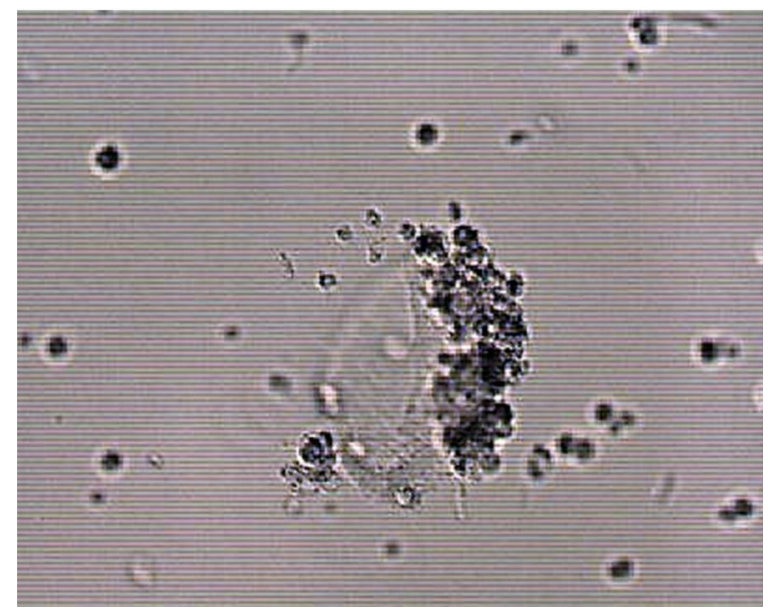

Fig. 1 A degenerated oocyte (DEG) at ovum pick-up (OPU)

in accordance to specific guidelines for: pronuclei (PN) assessment, PN fading, time ( $\mathrm{t}$ ) to $2,3,4,5$ and 8 cells. Additional kinetic markers and an Alfa ESHRE score, as well as the common morphology grade are also used for evaluation of the embryos [10].

The presence of DEG oocyte in the cohort of aspirated oocytes reduces the number of oocytes available for fertilization, whether they affect cycle outcomes is still questionable. To date, no study has evaluated the correlation between the presence of DEG oocyte at OPU and embryo morphokinetics. This study assessed morphokinetics of embryos cultured in a time-lapse incubator and compared cohorts with and without DEG oocytes.

\section{Material and methods}

This retrospective cohort study was conducted in a single reproductive centre. Records of all patients and their embryos were collected. The information of all embryos cultured in a time-lapse incubator from January 1, 2016 until September 31, 2017 were evaluated. To reflect the broad range of patients typically encountered in clinical practice, no inclusion/exclusion criteria were applied regarding baseline characteristics apart from the fact that no testicular sperm cycles were included. Cycles in which transfers were cancelled due to endometrial polyps, premature progesterone elevation and the use of donor oocyte were not included in the study. Institutional Review Board approval was obtained for this retrospective study.

The treatment protocol, type and doses of gonadotropins were prescribed on a case-by-case basis, based on patient characteristics and clinician preferences and judgment. The initial dose of gonadotropin was individualized for each patient according to age, basal follicle stimulating hormone (FSH) levels, antral follicle count, body mass index (BMI), and previous response to ovarian stimulation. Three main protocols were included in the study: long agonist, short flare and antagonist. Patients underwent controlled ovarian stimulation by recombinant follicle stimulating hormone $(\mathrm{rFSH})$ alone (Gonal-F, Merck-Serono; or Puregon, MSD); highly purified human menopausal gonadotropin (HPhMG) alone (Menopur, Ferring Pharmaceutical); or $\mathrm{rFSH}$ combined with HPhMG. All treatments were conducted as previously described [11-14]. Estrogen and progesterone levels were measured at every follow-up visit, including the day of human chorionic gonadotropin (hCG) (Ovitrelle MerckSerono) administration, before egg retrieval. hCG was administered for final maturation of oocytes when at least three mature $(>17 \mathrm{~mm}$ ) follicles were identified by transvaginal scan, combined with appropriate peripheral serum E2 levels. Oocytes were aspirated approximately $36 \mathrm{~h}$ after hCG injection. For luteal phase support, patients received $300 \mathrm{mg}$ micronized progesterone (Endometrin ${ }^{\oplus}$, Ferring, Israel) in three divided doses daily. Two different aspiration needles were routinely used 17G/35mm (Cook Medical $^{\mathrm{Tm}}$, Bloomington, IN, USA) and 20-17G/35mm (Sense ${ }^{\mathrm{T \mu}}$, Vitrolife Sweden AB, Gothenburg, Sweden).

After oocyte retrieval, IVF or ICSI was performed. After ICSI, the injected oocytes were placed on EmbryoSlides with one-step medium with SPS (SAGE; Origio) and incubated in the automated time-lapse EmbryoScope ${ }^{\mathrm{rm}}$ (Unisense FertiliTech, Aarhus, Denmark) up to 5 days with $5.8 \% \mathrm{CO} 2$ at $37.0{ }^{\circ} \mathrm{C}$ and $5 \% \mathrm{O} 2$. Using embryo viewer software Images of each embryo were acquired every 10 min in 7 focal planes, starting from the second polar body extraction up to $120 \mathrm{~h}$ after fertilization, to determine the exact timing of cell divisions [8]. They received a Known Implantation Data score (KID) [9], and Alfa ESHRE score, as well as the common morphology grade [10]. A maximum of two embryos were transferred on day 3 or one on day 5 of embryo development. The remaining top- 
quality embryos were vitrified and used in the next frozen embryo transfer, if no pregnancy was achieved in the fresh cycle. Embryo quality was evaluated as well, on the day of transfer according to number of cells, symmetry, granularity, type, percentage of fragmentation, presence of multinucleate blastomers, and degree of compaction, as previously described [15]. A top-quality embryo included the following parameters: $4-5$ cells on day 2 or $3 ;>6$ equal-sized blastomeres and $\leq 20 \%$ fragmentation; no multinucleate cells and KID score and Alfa ESHRE score of 5, 3 or 5,2 or 4,3 or 4,2 , respectively.

Data collection included baseline parameters (age, parity, BMI, number of previous IVF/ICSI cycles, basal FSH), cycle characteristics (length of follicular phase, amount of gonadotropins used, endometrial thickness and estradiol levels on day of hCG administration) and cycle outcomes (number of oocytes retrieved, fertilization and cleavage rates, number of top-quality embryos, and whether clinical pregnancy occurred).

$\beta$-hCG test was measured 14 days after embryo transfer, and the clinical pregnancy and implantation rates were confirmed when a gestational sac with fetal heart beat was visible by ultrasound examination after 6 weeks of pregnancy. Demographic data, treatment information and results, and pregnancy outcome were recorded and followed until delivery.

\section{Statistical analysis}

Statistical analysis was performed using the SPSS software package (SPSS Inc., Chicago, IL). We used Shapiro Wilks test to evaluate the distribution of the data. Comparisons were analyzed using Student's t test or MannWhitney U test, when appropriate. Proportions were compared using Chi-square test or Fisher exact test. $P$ value less than 0.05 was considered significant. We used multivariate logistic regression analysis to test all possible factors that may contribute to the occurrence or presence of DEG oocyte in the cohort of aspirated oocytes, and thus might have influenced clinical results.

\section{Results}

A total of 399 IVF/ICSI cycles and 2980 embryos were evaluated. In 81 of 399 cycles at least one DEG oocyte was observed at the time of OPU (DEG GROUP). The remaining 318 cycles without presence of DEG oocyte at OPU (NON DEG GROUP) were compared as a control group. Table 1 presents baseline patient characteristics of both groups.

Tables 2 and 3 show treatment parameters and outcomes comparing the DEG and NON DEG groups. In the DEG group, significantly more oocytes were collected per patient $(12.9 \pm 7.2$ vs. $10.1 \pm 6.1, P<0.001)$ and a trend towards a higher number of mature oocytes was seen $(7.3 \pm 4.4$ vs. $8.4 \pm 4.9 P=0.063)$. A significantly higher serum estradiol level on hCG trigger day (1638 \pm $798 \mathrm{pg} / \mathrm{ml}$ vs. $1990 \pm 1304, P=0.002$ ) was found in the DEG GROUP. No difference was found in fertilization rates. Morphokinetics and developmental scores of the embryos were significantly worse in the DEG GROUP, (KID $3.4 \pm 1.6$ vs. $3.2 \pm 1.6 \mathrm{P}=0.002$ and ESHRE $1.5 \pm 1.1$ vs. $1.4 \pm 1.0 P=0.046)$. Importantly, significantly more patients achieved top-quality embryos in the NON DEG group $(58.8 \%$ vs. $53.0 \%, P=0.03)$. Pregnancy rate and clinical pregnancy rate were not affected and were comparable between the two groups (Table 2).

A multivariate analysis was conducted to test all possible factors that may contribute to the presence of DEG oocyte in the cohort (Table 3). Maternal age, BMI, simulation protocols and type of needle used for OPU were included in the analysis. Significant predictors for the presence of a DEG oocyte at OPU were Stimulation protocols using decapeptyl (Flare and Long) compared with Antagonist protocol (Flare protocol: odds ratio $(\mathrm{OR}=$ 3.25, 95\%CI-1.39-7.60, $P=0.007)$ and Long down

Table 1 Patient characteristics

\begin{tabular}{|c|c|c|c|}
\hline Characteristic & Non DEG group $(n=318)$ & DEG group $(n=81)$ & $P$-value \\
\hline Age (years) (mean \pm STDV) & $35.1 \pm 5.9$ & $34.4 \pm 5.8$ & NS \\
\hline BMI (mean \pm STDV) & $25.1 \pm 5.5$ & $25.9 \pm 5.6$ & NS \\
\hline \multicolumn{4}{|l|}{ Etiology of infertility } \\
\hline Age/Unexplained/single & $117(36.8 \%)$ & $34(45.7 \%)$ & NS \\
\hline PCOS/Anovulation & $13(4.1 \%)$ & $4(4.9 \%)$ & NS \\
\hline Male factor & $111(34.9 \%)$ & $31(38.3 \%)$ & NS \\
\hline Mechanical/Endometriosis & $40(12.6 \%)$ & $5(6.2 \%)$ & NS \\
\hline Combined & $37(11.6 \%)$ & $4(4.9 \%)$ & NS \\
\hline LH & $5.9 \pm 2.7$ & $5.8 \pm 2.6$ & NS \\
\hline FSH & $8.5 \pm 2.7$ & $7.6 \pm 2.7$ & NS \\
\hline E2 & $70.3 \pm 78.9 ; 44[29.5-71.5]$ & $72.7 \pm 65.0 ; 50[39.5-89.5]$ & NS \\
\hline
\end{tabular}

DEG oocyte Degenerated oocyte, BMI Body mass index, PCOS Polycystic ovary syndrome, LH Luteinizing hormone, FSH Follicle stimulating hormone 
Table 2 Cycle characteristics and outcomes

\begin{tabular}{|c|c|c|c|}
\hline Characteristic & Non DEG group $(n=318)$ & DEG group $(n=81)$ & $P$-value \\
\hline Estradiol on hCG trigger day (pgr/dl) & $1638 \pm 798$ & $1990 \pm 1304$ & 0.002 \\
\hline Progesterone level at hCG trigger day & $0.67 \pm 0.45$ & $0.74 \pm 0.47$ & NS \\
\hline Endometrium (mm) & $9.7 \pm 2.3$ & $9.6 \pm 2.2$ & NS \\
\hline Duration of treatment (days) & $9.9 \pm 2.6$ & $10.5 \pm 2.6$ & NS \\
\hline \multicolumn{4}{|l|}{ Protocol Number/total cycles (\%) } \\
\hline Long Protocol & $51(16.2)$ & $21(27.6)$ & 0.03 \\
\hline Flare/Short agonist & $26(8.3)$ & $11(14.5)$ & NS \\
\hline Antagonist & $233(74)$ & $44(57.9)$ & 0.007 \\
\hline Modified natural cycle & $4(1.3 \%)$ & 0 & NS \\
\hline \multicolumn{4}{|l|}{ Treatment outcome } \\
\hline Number of Oocyte collected & $10.1 \pm 6.1$ & $12.9 \pm 7.2$ & $P<0.001$ \\
\hline M2 & $7.3 \pm 4.4$ & $8.4 \pm 4.9$ & $P=0.063$ \\
\hline 2PN & $5.8 \pm 3.8$ & $6.2 \pm 3.9$ & NS \\
\hline KID & $3.4 \pm 1.6$ & $3.2 \pm 1.6$ & $P=0.002$ \\
\hline ESHRE & $1.5 \pm 1.1$ & $1.4 \pm 1.0$ & $P=0.046$ \\
\hline $\mathrm{KID}=4+5$ (Top quality embryo) & $1174(58.8 \%)$ & $314(53.0 \%)$ & $P=0.012$ \\
\hline ESHRE $2+3$ (Top quality embryo) & $1041(52.2 \%)$ & $282(47.6 \%)$ & $P=0.049$ \\
\hline \multicolumn{4}{|l|}{ 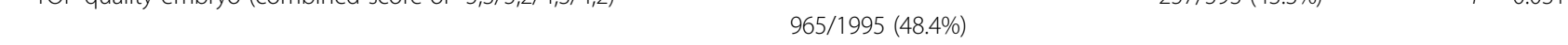 } \\
\hline Chemical pregnancy/cycle & $105 / 318(38.5 \%)$ & $32(39.5 \%)$ & NS \\
\hline Clinical pregnancy/cycle & $105 / 318(38.5 \%)$ & $29(35.8 \%)$ & NS \\
\hline
\end{tabular}

DEG Degenerated oocyte, $h C G$ Human chorionic gonadotropin

regulation protocol: $\mathrm{OR}=2.60,95 \% \mathrm{CI}=1.34-5.04, P=$ $0.005)$. The chance for DEG was higher when OPU was conducted with 17-20G needle compared with the $17 \mathrm{G}$ needle $(\mathrm{OR}=2.035,95 \% \mathrm{CI}=1.115-3.61, P=$ 0.015).

In analyses of the DEG group for the prevalence of DEG oocytes in the cohort at OPU, we found that a lower proportion of DEG in the cohort resulted in a higher pregnancy rate $(\mathrm{OR}=0.11,95 \% \mathrm{CI} 0.02-0.56$, $P=0.008)$. The ratio of DEG oocytes per cycle was negatively correlated with pregnancy rate. In the group of DEG oocytes, we found that OPU conducted with $17 \mathrm{G}$ needle resulted in a higher pregnancy rate as compared with $17-20 \mathrm{G}$ needle $(47.8 \%$ vs. $23.8 \%$; $P<0.0001)$.

Table 3 Multivariate analysis to predict DEG oocytes in the cohort

\begin{tabular}{lllll}
\hline Woman's age & Odds ratio & \multicolumn{2}{c}{$95 \% \mathrm{Cl}$} & $\boldsymbol{P}$-value \\
\cline { 3 - 4 } & 0.980 & 0.932 & 1.029 & 0.415 \\
\hline BMl & 0.967 & 0.920 & 1.016 & 0.188 \\
Long protocol & 2.604 & 1.344 & 5.042 & 0.005 \\
Flare protocol & 3.250 & 1.389 & 7.605 & 0.007 \\
Needle type & 2.035 & 1.146 & 3.613 & 0.015 \\
\hline
\end{tabular}

DEG oocyte Degenerated oocyte, BMI Body mass index

\section{Discussion}

The current study evaluated the effect of degenerated oocytes (DEG) found at OPU on clinical outcomes, including cycle outcome and embryo morphokinetics comparing the presence and absence of DEG oocyte in the cohort of aspirated oocytes. This is the largest study to account and evaluate the presence of DEG at OPU. Our results revealed that the impact of the stimulation protocol and the size of needle used for OPU had significant influence on presence of DEG oocyte in the cohort at OPU.

Based on previous studies, the presence of DEG in the cohort of aspirated oocytes can be the result of physical and mechanical forces contributed by needle bevel, aspiration vacuum pressure [16], technique of OPU including scraping of the follicle and needle type [15, 17]. Additional factors are the intrinsic oocyte quality, which can be influenced by infertility cause, obesity and stimulation protocol $[2,5,18-20]$.

The cause of DEG oocyte and its impact at OPU are only minimally discussed in the literature. Cohen et al. [16] showed that manual aspiration by a syringe resulted in a higher rate of damaged oocytes compared with mechanical aspiration by pump [16]. Oride et al. [7] hypothesized that the mechanical forces during pick up caused oocyte damage at OPU and had an impact on cycle outcome. They demonstrated that cycles with 
higher number of oocytes significantly correlated with increased presence of DEG oocytes, and the fertilization rate and cleavage rate of those cycles were significantly lower [7]. Cinar et al. [2] evaluated the impact of the ratio of DEG oocyte of the total aspirated oocytes per pick-up. They found that the ratio (DEG/total oocyte) increased when more oocytes were collected per cycle. This study, in agreement with our results, reported a negative correlation between the DEG ratio to fertilization rate and cleavage rate. Pregnancy rate was highest when no DEG were collected [2].

Our cohort included a group of 81 cycles $(20 \%)$ in which at least one DEG oocyte was found (Fig. 1). Possibly a causative correlation exists between the presence of DEG oocyte and the sheer stress applied along the needle. Animal models demonstrated the effect of aspiration pressure and needle size on damaged oocytes [21, 22]. It is well established that according to Hagen-Poiseuille's law [23], at the same pressure, the flow in a small gauge needle is slower than in larger gauge. Meaning that the Laminar flow within a needle shows a parabolic distribution due to different velocities of the fluid along the needle. Due to suction forces along the shaft, fluid moves slower close to the inner wall of the needle and faster in its center. In addition, the oocyte size is smaller than the needle diameter, and due to different shear stresses, the oocyte comlex may be bounced inside the lumen. It was shown that higher pressure and thicker needles can damage the cumulus-oocytecomplex and was suggested to have great effect on oocyte and embryo quality and reduce the blastulation rate $[16,17,21,24]$.
Human studies assessing oocyte quality report conflicting data regarding the impact of DEG oocyte on cycle outcome [3, 4, 19, 20, 25-29]. Lazzaroni-Tealdi et al. [6] use oocyte scoring to provide useful information on embryo quality and showed that oocyte score provided significantly greater predictive value for clinical pregnancy. Shi et al. [19] reported significantly lower fertilization rate, poorer embryo quality, implantation and clinical pregnancy rate in the group of patients that had at least one damaged zona pellucida in the cohort. We assumed that the same force that caused the DEG oocyte might influence the rest of the cohort and lead to the reduced fertilization, embryo quality and cycle results. We found a significant difference in embryo morphokinetics, with poorer embryo scoring in the DEG group, but without significant differences in implantation and clinical pregnancy rates. The comparable outcome between the groups might be due to compensation by a higher number of collected oocytes or the fact that eventually, a top-quality embryo was transferred.

Our study found that the needles used for OPU and the treatment protocol were associated with DEG oocyte at OPU. We used two different types of needles for oocyte aspiration, a $17 \mathrm{G}$ needle or a changing diameter 17$20 G$ needle. The vacuum pressure is different for each needle, $140 \mathrm{mmHg}$ for $17 \mathrm{G}$ and $120 \mathrm{mmHg}$ for the 17 20G (manufactory recommendation). In multivariate analysis, we found that using 17-20G needle was significantly correlated with a higher number of DEG oocytes at OPU and a significantly worse morphokinetics score.

\section{Pregnancy rate per percentage of DEG oocyte}

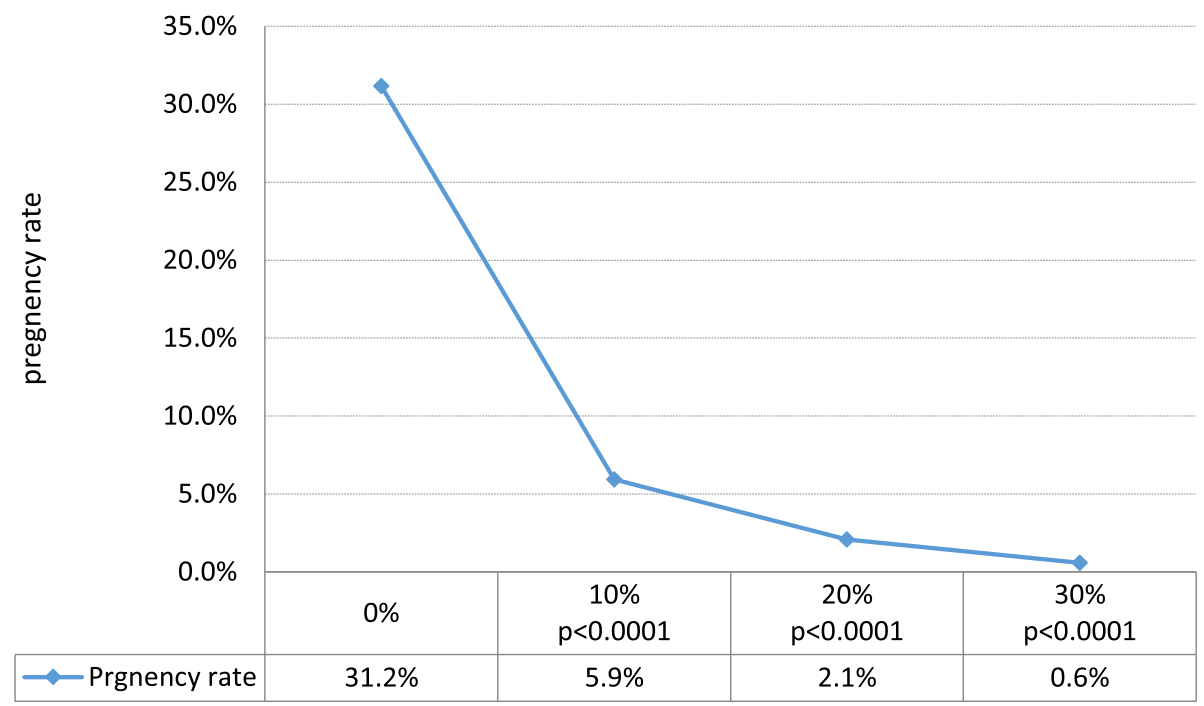

Percentage of DEG

Fig. 2 The impact of the prevalence of DEG oocyte on pregnancy outcome 
However, in agreement with Wikland et al. [30] our study did not show any difference between the two needles in pregnancy rates.

Stimulation protocol was also found to impact the presence of DEG. Protocols which used GnRH agonist including long down regulation protocol and flare protocol had twice and three times higher risk of DEG oocyte, respectively, as compared with antagonist protocol. In contrast to our results, Cinar et al. [2] reported that more damaged oocytes was correlated with the use of GnRH antagonist protocol.

The limitations of our study are inherent to its retrospective nature and to the patient treatment protocol and the fact that this was a heterogenic group with different causes of infertility. About 70\% of our patients start with antagonist protocol, only after failure of the antagonist protocol, we change the protocol to long down regulation or flare.

The strengths of our study are that it is one of the largest to report DEG oocyte at OPU, the impact on oocyte performance and the follow-up until pregnancy.

In conclusion, we found a negative correlation between the prevalence of DEG oocyte in the cohort to pregnancy rate. As the percentage of DEG oocytes increased, the pregnancy rate significantly decreased (Fig. 2). Another important finding was the association between the number of aspirated oocytes and the percentage of DEG. Taken together, these findings may reflect on the impact of the aspiration forces along the needle or the type of protocol used in the cohort of the DEG group. Further studies are needed to assess the influence of the protocols, the medication used and the needle type on DEG oocytes.

\section{Consent to participate}

Not relevant.

\section{Authors' contributions}

All authors contributed substantially to this work. The authors collectively developed the original concept of this study. YA and ESP wrote the manuscript. ESP and NA and OL revised it critically. Data collection was performed by NR., MM, DP, statistical analysis by ESP. All authors contributed to critical discussion and reviewed and approved the final version of the manuscript for submission.

\section{Funding}

No

\section{Availability of data and materials}

Yes

Ethics approval and consent to participate

Approved.

\section{Ethics approval}

Accepted.

\section{Consent for publication}

Approved.

\section{Competing interests}

No

Received: 4 September 2019 Accepted: 20 August 2020

Published online: 17 September 2020

\section{References}

1. Conti M, Franciosi F. Acquisition of oocyte competence to develop as an embryo: integrated nuclear and cytoplasmic events. Hum Reprod Update. 2018;24:245-66. https://doi.org/10.1093/humupd/dmx040.

2. Cinar O, Demir B, Dilbaz S, Saltek S, Aydin S, Goktolga U. Does empty zona pellucida indicate poor ovarian response on intra cytoplasmic sperm injection cycles? Gynecol Endocrinol. 2012;28:341-4. https://doi.org/10.3109/ 09513590.2011 .631632$.

3. Serhal PF, Ranieri DM, Kinis A, Marchant S, Davies M, Khadum IM. Oocyte morphology predicts outcome of intracytoplasmic sperm injection. Hum Reprod. 1997;12:1267-70. https://doi.org/10.1093/humrep/12.6.1267.

4. Xia P. Intracytoplasmic sperm injection: correlation of oocyte grade based on polar body, perivitelline space and cytoplasmic inclusions with fertilization rate and embryo quality. Hum Reprod. 1997;12:1750-5. https:// doi.org/10.1093/humrep/12.8.1750.

5. Rienzi L, Balaban B, Ebner T, Mandelbaum J. The oocyte. Hum Reprod. 2012; 27(Suppl 1):i2-21. https://doi.org/10.1093/humrep/des200.

6. Lazzaroni-Tealdi E, Barad DH, Albertini DF, Yu Y, Kushnir VA, Russell H, et al. Oocyte scoring enhances embryo-scoring in predicting pregnancy chances with IVF where it counts Most. PLoS One. 2015;10:e0143632. https://doi.org/ 10.1371/journal.pone.0143632.

7. Oride A, Kanasaki H, Hara T, Ohta H, Kyo S. Characterization of oocyte retrieval cycles with empty zona pellucida. Reprod Med Biol. 2018;17:71-6. https://doi.org/10.1002/rmb2.12071.

8. Meseguer M, Herrero J, Tejera A, Hilligsøe KM, Ramsing NB, Remohí J. The use of morphokinetics as a predictor of embryo implantation. Hum Reprod. 2011;26:2658-71. https://doi.org/10.1093/humrep/der256.

9. Petersen BM, Boel M, Montag M, Gardner DK. Development of a generally applicable morphokinetic algorithm capable of predicting the implantation potential of embryos transferred on Day 3. Hum Reprod. 2016;31:2231-44.

10. Alpha Scientists in Reproductive Medicine and ESHRE Special Interest Group of Embryology. The Istanbul consensus workshop on embryo assessment: proceedings of an expert meeting. Hum Reprod. 2011;26:1270-83. https:// doi.org/10.1093/humrep/der037.

11. Olivennes F, Alvarez S, Bouchard P, Fanchin R, Salat-Baroux J, Frydman R. The use of a GnRH antagonist (Cetrorelix) in a single dose protocol in IVFembryo transfer: a dose finding study of 3 versus 2 mg. Hum Reprod. 1998; 13:2411-4. https://doi.org/10.1093/humrep/13.9.2411.

12. Albano C, Smitz J, Camus M, Riethmüller-Winzen H, Van Steirteghem A, Devroey P. Comparison of different doses of gonadotropin-releasing hormone antagonist Cetrorelix during controlled ovarian hyperstimulation. Fertil Steril. 1997;67:917-22. https://doi.org/10.1016/s0015-0282(97)81407-0.

13. Huirne JAF, Lambalk CB, van Loenen ACD, Schats R, Hompes PGA, Fauser BCJM, et al. Contemporary pharmacological manipulation in assisted reproduction. Drugs. 2004;64:297-322. https://doi.org/10.2165/00003495200464030-00005.

14. Barbieri RL, Hornstein MD. Assisted reproduction-in vitro fertilization success is improved by ovarian stimulation with exogenous gonadotropins and pituitary suppression with gonadotropin-releasing hormone analogues. Endocr Rev. 1999;20:249-52. https://doi.org/10.1210/edrv.20.3.0363.

15. Rose Bl. Approaches to oocyte retrieval for advanced reproductive technology cycles planning to utilize in vitro maturation: a review of the many choices to be made. J Assist Reprod Genet. 2014;31:1409-19. https:// doi.org/10.1007/s10815-014-0334-9.

16. Cohen J, Avery S, Campbell S, Mason BA, Riddle A, Sharma V. Follicular aspiration using a syringe suction system may damage the zona pellucida. J In Vitro Fert Embryo Transf. 1986;3:224-6.

17. Horne R, Bishop CJ, Reeves G, Wood C, Kovacs GT. Aspiration of oocytes for in-vitro fertilization. Hum Reprod Update. 1996;2:77-85. https://doi.org/10. 1093/humupd/2.1.77.

18. Shah DK, Missmer SA, Berry KF, Racowsky C, Ginsburg ES. Effect of obesity on oocyte and embryo quality in women undergoing in vitro fertilization. Obstet Gynecol. 2011;118:63-70. https://doi.org/10.1097/AOG. Ob013e31821fd360. 
19. Shi W, Xu B, Wu L-M, Jin R-T, Luan H-B, Luo L-H, et al. Oocytes with a dark zona pellucida demonstrate lower fertilization, implantation and clinical pregnancy rates in IVF/ICSI cycles. PLoS One. 2014;9:e89409. https://doi.org/ 10.1371/journal.pone.0089409.

20. Atzmon Y, Shoshan-Karchovsky E, Michaeli M, Aslih N, Shrem G, Ellenbogen A, et al. Obesity results with smaller oocyte in in vitro fertilization/ intracytoplasmic sperm injection cycles-a prospective study. J Assist Reprod Genet. 2017;34:1145-51. https://doi.org/10.1007/s10815-017-0975-6.

21. Bols PE, Van Soom A, Ysebaert MT, Vandenheede JM, de Kruif A. Effects of aspiration vacuum and needle diameter on cumulus oocyte complex morphology and developmental capacity of bovine oocytes. Theriogenology. 1996;45:1001-14. https://doi.org/10.1016/0093-691x(96)00028-3.

22. Fry RC, Niall EM, Simpson TL, Squires TJ, Reynolds J. The collection of oocytes from bovine ovaries. Theriogenology. 1997;47:977-87. https://doi. org/10.1016/s0093-691x(97)00054-x.

23. Sutera SP, Skalak R. The history of Poiseuille law. Annu Rev Fluid Mech. 1993; 25(1):1-20.

24. Kumaran A, Narayan PK, Pai PJ, Ramachandran A, Mathews B, Adiga SK. Oocyte retrieval at $140-\mathrm{mmHg}$ negative aspiration pressure: a promising alternative to flushing and aspiration in assisted reproduction in women with low ovarian reserve. J Hum Reprod Sci. 2015;8:98-102. https://doi.org/ 10.4103/0974-1208.158617.

25. Durinzi KL, Saniga EM, Lanzendorf SE. The relationship between size and maturation in vitro in the unstimulated human oocyte. Fertil Steril. 1995;63: 404-6. https://doi.org/10.1016/50015-0282(16)57377-4.

26. Romão GS, MCPM A, de Melo AS, de Albuquerque Salles Navarro PA, Ferriani RA, Dos Reis RM. Oocyte diameter as a predictor of fertilization and embryo quality in assisted reproduction cycles. Fertil Steril. 2010;93:621-5. https://doi.org/10.1016/j.fertnstert.2008.12.124.

27. Rienzi L, Vajta G, Ubaldi F. Predictive value of oocyte morphology in human IVF: a systematic review of the literature. Hum Reprod Update. 2011;17:3445. https://doi.org/10.1093/humupd/dmq029.

28. Ebner T, Moser M, Tews $\mathrm{G}$. Is oocyte morphology prognostic of embryo developmental potential after ICSI? Reprod BioMed Online. 2006;12:507-12. https://doi.org/10.1016/s1472-6483(10)62006-8.

29. Loutradis D, Drakakis P, Kallianidis K, Milingos S, Dendrinos S, Michalas S. Oocyte morphology correlates with embryo quality and pregnancy rate after intracytoplasmic sperm injection. Fertil Steril. 1999;72:240-4. https://doi. org/10.1016/s0015-0282(99)00233-2

30. Wikland M, Blad S, Bungum L, Hillensjö T, Karlström PO, Nilsson S. A randomized controlled study comparing pain experience between a newly designed needle with a thin tip and a standard needle for oocyte aspiration. Hum Reprod. 2011;26:1377-83. https://doi.org/10.1093/humrep/der100.

\section{Publisher's Note}

Springer Nature remains neutral with regard to jurisdictional claims in published maps and institutional affiliations.

Ready to submit your research? Choose BMC and benefit from:

- fast, convenient online submission

- thorough peer review by experienced researchers in your field

- rapid publication on acceptance

- support for research data, including large and complex data types

- gold Open Access which fosters wider collaboration and increased citations

- maximum visibility for your research: over $100 \mathrm{M}$ website views per year

At $\mathrm{BMC}$, research is always in progress.

Learn more biomedcentral.com/submissions 